\title{
Propriétés Physiques et Mécaniques des Graines et Amandes de Jatropha curcas $L$.
}

\author{
Danebe, K. A., \\ Département de Génie Textile et Cuir, Ecole Nationale Supérieure \\ Polytechnique, Université de Maroua, Cameroun
}

Djeumako, B.,

Département de Génie Mécanique, Ecole Nationale Supérieure Des Sciences

Agro-Industrielles, Université de Ngaoundéré, Cameroun

Beda, T.,

Département de Physique, Faculté des Sciences,

Université de Ngaoundéré, Cameroun

Samon, J. B.,

Département de Génie Mécanique, Ecole Nationale Supérieure Des Sciences

Agro-Industrielles, Université de Ngaoundéré, Cameroun

Doi:10.19044/esj.2019.v15n36p81 ～URL:http://dx.doi.org/10.19044/esj.2019.v15n36p81

\section{Résumé}

Cette étude a déterminé les propriétés physiques et mécaniques des graines et amandes de Jatropha curcas L. (Jatropha), originaire de l'ExtrêmeNord du Cameroun. Les paramètres physiques tels que les dimensions moyennes, les caractéristiques massiques, les paramètres de forme et les caractéristiques volumiques ont été évalués. La raideur de la coque a été définie en variant la vitesse de compression de la machine d'essai de traction universel EZ 50. A grande vitesse de compression $(5-40 \mathrm{~mm} / \mathrm{mn})$, les brisures d'amandes et de coques qui ont été observées ont été représentées sur les courbes de compression des graines par plusieurs points de rupture. Avec une faible vitesse de l'ordre de $1 \mathrm{~mm} / \mathrm{mn}$, les courbes de compression ont été linéaires. La valeur moyenne de la force de rupture en compression $\mathrm{F}_{\mathrm{r}}(\mathrm{N})$ et la raideur $\mathrm{K}(\mathrm{N} / \mathrm{mm})$ des coques calculées ont été respectivement 134,41 N et $105 \mathrm{~N} / \mathrm{mm}$. Les essais d'extraction d'huile de Jatropha sont réalisés à température ambiante $\left(23-24^{\circ} \mathrm{C}\right)$ avec des charges comprises entre 1000 et $12000 \mathrm{~N}$. Ce qui a permis d'obtenir les relations mathématiques entre la force de compression et la déformation relative : $\mathrm{F}(\Delta \mathrm{l})=8,91 \cdot \operatorname{Exp}(0,94 \cdot \Delta \mathrm{l})-9,41$ (N); entre la contrainte de compression et la déformation relative : $\sigma(\varepsilon)=0,08 \cdot \operatorname{Exp}(6,89 \cdot \varepsilon)-0,08(\mathrm{MPa})$. L'algorithme de « Trust region » et de " Levenberg-Marquardt », ont été respectivement intégré dans l'application MATLAB R2018a pour obtenir les modèles précédents. Les points limites 
pour le pressage des amandes sont compris entre 0,40 et 0,85 permettant d'obtenir les limites pour une extraction optimale de l'huile. La force de compression a été comprise entre $72,71 \mathrm{~N}$ et $4905,70 \mathrm{~N}$, pendant que la contrainte de compression varie entre 0,65 et $37,01 \mathrm{MPa}$ pour une consommation d'énergie allant de 0,13 à $2,83 \mathrm{~J} / \mathrm{mm}^{3}$

Mots clés: Jatropha curcas L., force de compression, contrainte de compression, vitesse de compression

\title{
Physicomechanical Properties of Jatropha Curcas L. Seeds and kernel.
}

\author{
Danebe, K. A.,
}

Département de Génie Textile et Cuir, Ecole Nationale Supérieure

Polytechnique, Université de Maroua, Cameroun

Djeumako, B.,

Département de Génie Mécanique, Ecole Nationale Supérieure Des Sciences

Agro-Industrielles, Université de Ngaoundéré, Cameroun

Beda, $T$.,

Département de Physique, Faculté des Sciences,

Université de Ngaoundéré, Cameroun

Samon, J. B.,

Département de Génie Mécanique, Ecole Nationale Supérieure Des Sciences

Agro-Industrielles, Université de Ngaoundéré, Cameroun

\begin{abstract}
This study has estimated physicomechanical properties of Jatropha curcas L. (Jatropha) seeds and kernels from the Far North Region of Cameroon. Physical parameters as mean sizes, mass characteristics, morphologic and volume characteristics were evaluated. The stiffness of the husk was estimated by varying the velocity of the universal tensile testing machine EZ 50. For a high compression velocity, $(5-40 \mathrm{~mm} / \mathrm{mn})$, compression diagrams of seeds showed several breaking point, we observed broken seeds and kernels with low velocity of $1 \mathrm{~mm} / \mathrm{mn}$, compression diagram are linear. The means value of the compression force $\operatorname{Fr}(\mathrm{N})$ and husk stiffness $\mathrm{K}(\mathrm{N} / \mathrm{mm})$ are respectively $134.41 \mathrm{~N}$ and $105 \mathrm{~N} / \mathrm{mm}$. Tensile tests of Jatropha oil were realized at $23-24^{\circ} \mathrm{C}$ with compression forces of 1000 and $12000 \mathrm{~N}$. We obtained mathematical relations between relative deformation and
\end{abstract}


compression force: $\mathrm{F}(\Delta \mathrm{l})=8.91 \cdot \operatorname{Exp}(0.94 \cdot \Delta \mathrm{l})-9.41(\mathrm{~N})$, compression stress and relative deformation: $\sigma(\varepsilon)=0.08 \cdot \operatorname{Exp}(6.89 \cdot \varepsilon)-0.08(\mathrm{MPa})$, using respectively the "trust region" and "Levenberg-Marquardt" algorithm related to MATLAB R 2018a software. The endpoints for and optimal extraction of Jatropha oil were 0.40 and 0.85 when the compression force were between $72.71 \mathrm{~N}$ and $4905.5 \mathrm{~N}$, the stress compression vavy between 0.65 and 37.01 $\mathrm{MPa}$, therefore the consumed energy varied between 0.13 and $3.83 \mathrm{~J} / \mathrm{mm}^{3}$.

Keywords: Jatropha Curcas L., Compression Force, Stress of Compression, Compression Velocity

\section{Introduction}

L'humanité est dépendante des ressources fossiles non seulement pour des besoins énergétiques, mais aussi elles servent de matière première pour l'approvisionnement des industries (Bagan et al., 2012). Cependant, le coût de plus en plus élevé de l'énergie fossile et la perspective d'un épuisement programmé de cette source énergétique naturelle non renouvelable ont été relevé. De plus, les phénomènes de changements climatiques imputables aux émissions des gaz à effet de serre (GES) dans l'atmosphère, ont amené à réfléchir aux développements de nouvelles sources d'énergie écologiquement propre et économiquement rentable (Shivani et al., 2011). C'est notamment le cas des énergies renouvelables avec le développement de la problématique des biocarburants comme solution palliative.

Plusieurs essences ont déjà été utilisées pour la production du biocarburant : les céréales, le sucre, les huiles végétales (Ballerini et Alazardtoux, 2006, Daudin et al., 2012). Cependant, cette production de biocarburant à partir des denrées alimentaires impacte négativement la sécurité alimentaire (Tangermann, 2007). En effet, dans son rapport, la FAO (2011) a indiqué que la flambée de la demande de matières premières agricoles pour la fabrication de biocarburants a contribué à une hausse des prix des denrées alimentaires, menaçant la sécurité alimentaire des plus démunis dans les zones urbaines et rurales. De plus, l'on assiste à des conflits liés à l'exploitation des espaces alloués aux cultures destinées à des essences pour biocarburant et ceux destinés à l'alimentation.

Il devenait donc impératif de trouver des essences agricoles qui permettent de gérer le problème de l'alimentation et du foncier. Des recherches menées dans ce sens ont révélé Jatropha curcas L. (Communément appelé Jatropha) comme étant une oléagineuse non alimentaire adaptée à la production du biocarburant ayant moins d'impact sur le foncier et n'entre pas en compétition avec les cultures alimentaires (Hartlieb et Gorriz, 2004).

En fait, l'huile de Jatropha a des propriétés physicochimiques proches de celle du diesel d'origine fossile. Des essais de cette huile ont déjà été 
effectués avec succès dans des moteurs diesel, des groupes pour éclairage et des réacteurs d'avion (Fotseu et al., 2011 ; Sidibé, 2011). Cependant, la production de cette huile n'est pas aisée en rapport avec le contexte sociotechnique des pays africains. Des presses à huile avec une capacité d'extraction de 10 à $30 \%$ existent sur le marché international avec des coûts assez élevés variant de $1450 €$ à $222000 €$. (Domergue et Pirot, 2008). De plus, la maintenance de ces équipements est très onéreuse.

Compte tenu du contexte et des compétences non négligeables dans la fabrication mécanique, des travaux sur la fabrication des équipements de l'unité de production de l'huile de Jatropha ont été initiés. Elles ont porté sur l'extraction mécanique notamment l'équipement de vannage (Djeumako et al., 2015).

Dans la suite, il est question de s'intéresser à l'unité d'extraction proprement dite et aux caractéristiques physiques des graines de Jatropha.

Ce travail a pour objectif de caractériser les graines et les amandes de Jatropha curcas L. de la Région de l'Extrême-Nord du Cameroun d'une part et d'avoir des données d'entrées importantes pour la conception et le dimensionnement d'un système de décorticage puis d'une presse à huile d'autre part. Cela permettrait de compléter l'unité de production de l'huile de Jatropha curcas L.

\section{Matériel et méthodes}

Matériel végétal

Le matériel végétal utilisé dans cette étude est constitué de graines de Jatropha curcas L. Ces graines ont été récoltées dans la Région de l'ExtrêmeNord du Cameroun ; plus précisément dans la ville de Maroua. Les graines ont été conditionnées dans un espace ouvert à température ambiante. La figure 1 présente des amandes et des graines de Jatropha.

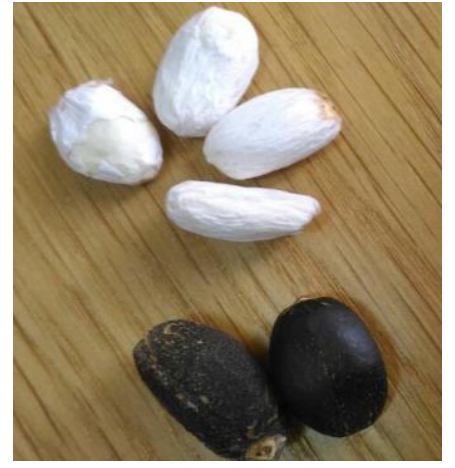

Figure 1 : Graines et amandes de Jatropha 


\section{Matériel technique}

Les essais de compression et d'extraction ont été réalisés avec la machine d'essais universelle EZ 50. La machine comporte les pilotes pour l'acquisition des données et elle est assistée par un ordinateur (Figure 2). Cet équipement provient du laboratoire de génie mécanique de la faculté d'ingénierie de l'université Vasile Alexandrii de Bacau - Roumanie. Les mesures des forces de compressions ont été effectuées selon la norme EN ISO 7500 (2004) avec une capacité nominale de $50 \mathrm{kN}$ et la précision de la mesure de force est de $+0,5 \%$ (à partir de $2 \%$ de la capacité du capteur).

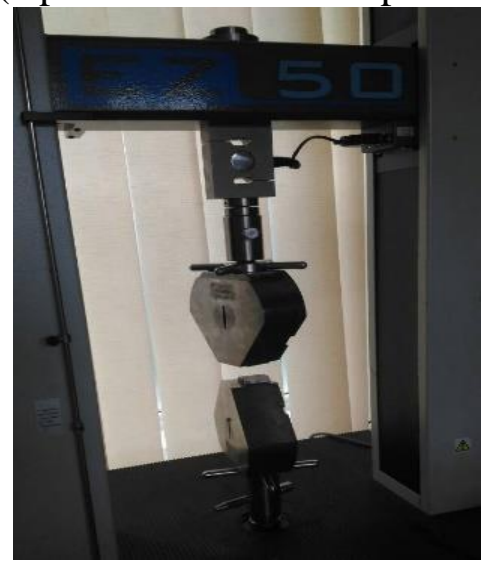

Figure 1: Machine d'essai universelle EZ 50.

\section{Méthodes}

\section{Détermination des propriétés physiques des graines et amandes de Jatropha}

Un lot de 100 graines a été utilisé pour mesurer la longueur, la largeur et l'épaisseur de chacune des graines à l'aide d'un pied à coulisse numérique dont la plage de mesure est comprise entre $0-150 \mathrm{~mm}$ et avec une précision : $\mathrm{de}+0,02 \mathrm{~mm} / 0,001(<100 \mathrm{~mm})$, et $+0,03 \mathrm{~mm} / 0,001(>100-150 \mathrm{~mm})$, (Figure 3).


Figure 2: Mesure des dimensions caractéristiques des graines avec un pied à coulisse numérique. $L, l$ et $e(\mathrm{~mm})$ représentent respectivement la longueur, la largeur et l'épaisseur de la graine (Djeumako et al., 2015)

La teneur en eau des graines a été déterminée suivant la norme ISO662-1998. 100 graines ont été écrasées à l'aide d'un moulin puis $5 \mathrm{~g}$ de moulure ont été séchés à l'étuve à $105^{\circ} \mathrm{C}$ pendant $24 \mathrm{~h}$ jusqu'à l'obtention de 
la masse constante. La teneur en eau en base sèche (bs) a été calculée selon la formule (1) :

$$
B_{(b s)}=\frac{\left(m_{o}-m\right)}{m_{0}} \times 100 \%
$$

$\mathrm{m}_{0}$ et $\mathrm{m}$ sont respectivement les masses initiales (en $\mathrm{g}$ ) de l'échantillon humide et de l'échantillon après évaporation.

La connaissance des dimensions axiales des graines permet de déterminer les caractéristiques des graines à l'aide des relations mathématiques (2) à (6), (Zavrajnov et Nikolow, 1990).

Elongation des graines $(\mathrm{E})$ :

$$
E=\frac{L}{l}
$$

Degré d'aplatissement des graines $(\mathrm{A})$ :

$$
A=\frac{l}{e}
$$

Diamètre moyen arithmétique des dimensions $\left(\mathrm{D}_{\mathrm{a}}\right)$ :

$$
D_{a}=\frac{(L+l+e)}{3}(\mathrm{~mm})
$$

Diamètre moyen géométrique $\left(\mathrm{D}_{\mathrm{g}}\right)$, (Calisir et al., 2005) :

$$
D_{g}=(L \times l \times e)^{\frac{1}{3}}(\mathrm{~mm})
$$

Sphéricité des graines $(\mathrm{S})$ :

$$
S=L^{-1} \times(L \times l \times e)^{\frac{1}{3}}
$$

Dans les formules précédentes, « $\mathrm{L}$ » représente la longueur, « 1 » la largeur, et «e » l'épaisseur.

Le pycnomètre de Brucker a permis d'évaluer le volume réel $\left(V_{o}\right)$ des amandes et des graines. A cet effet, la masse $\left(m_{o}\right)$ de 1000 graines et amandes de Jatropha a été obtenue par pesée à l'aide d'une balance électronique de précision $0,001 \mathrm{~g}$ selon la norme ISO-520.

La mesure a été effectuée par prises d'essai de 2 lots de 50 graines et 50 amandes. La masse volumique réelle $\rho$ a ensuite été déduite :

$$
\rho=m_{0} / V_{0}\left(\mathrm{~kg} / \mathrm{m}^{3}\right)
$$

Le volume des 1000 graines (ou amandes) a permis d'estimer le volume moyen $\mathrm{V}_{\mathrm{z}}$ d'une graine ou d'une amande. Le volume $\mathrm{V}_{\mathrm{z}}$ couplé avec la masse volumique $(\rho)$, ont fourni les paramètres massiques des graines ou des amandes définis par les relations (8), (9) et (10) (Razavi et al., 2007 ; Chasseray, 1991).

Diamètre équivalent $\left(D_{E}\right)$ :

$$
D_{E}=1.24 \sqrt[3]{V_{z}}(m)
$$

Aire massique $\left(S_{m}\right)$ : 


$$
S_{m}=6 \times\left(\rho \times D_{E}\right)^{-1}\left(m^{2} / k g\right)
$$

Volume massique $(S$ )

$$
S=6 \times \rho^{-1}\left(\mathrm{~m}^{3} / \mathrm{kg}\right)
$$

La masse volumique apparente des graines ou des amandes $\left(\rho_{\mathrm{a}}\right)$ a été obtenue à partir de la masse des graines ou des amandes dans un récipient de volume connu avec les espaces vides inter granulaires naturels.

La porosité $(\mathrm{P})$ ou degré d'espace vide contenu dans le produit a été défini en pourcentage $(\%)$, lorsque celle-ci n'est soumise à aucune pression effective extérieure. Elle a été obtenue par la relation mathématique (11) (Mohsenin, 1986) :

$$
P=100 \times\left(\rho-\rho_{a}\right) / \rho_{a}(\%)
$$

Le coefficient de frottement statique de la graine a été déterminé à partir de son angle maximal au repos. L'angle de repos est la caractéristique de la matière qui indique la cohésion entre les unités individuelles de la matière (Sirisomboon et al., 2007). Il a été déterminé en utilisant les méthodes de remplissage et de vidage (Amin et al., 2004 ; Bart-Plange et Baryeh, 2004). Coefficient de frottement statique

$$
\mu=\tan \theta
$$

Angle statique au repos (Orguven et Kubilay, 2004) :

$$
\theta=\tan ^{-1}\left(\frac{2 H}{D}\right)
$$

Avec $\mathrm{H}(\mathrm{mm})$ la hauteur et $\mathrm{D}(\mathrm{mm})$ le diamètre du cône formés par les graines.

Les données tels que les teneurs en eau, les dimensions, les volumes, les masses, les densités, les masses volumiques réelles et apparentes sont collectées en 4 répétitions. La comparaison des moyennes des diamètres a été réalisée par le test de Tukey avec l'analyse de variance (ANOVA).

\section{Détermination des propriétés mécaniques des graines de Jatropha curcas L.}

Les essais de compression ont été effectués sur un échantillon de 25 graines de Jatropha curcas $L$. à des vitesses de 1,$5 ; 10,20$ et $40 \mathrm{~mm} / \mathrm{mn}$.

La charge de rupture $\mathrm{F}_{\mathrm{Rmax}}(\mathrm{N})$ est la charge qu'il faut appliquer pour briser la coque de la graine de Jatropha (Figure 4). La déformation au point de rupture $\mathrm{D}_{\mathrm{Rmax}}(\mathrm{mm})$, est définie comme suivant la direction de chargement. Le taux de déformation au point de rupture $\mathrm{R}_{\mathrm{DR}}$, quant à lui, est la déformation axiale au point de rupture de l'échantillon. Il est le rapport de la déformation au point de rupture à la dimension de l'échantillon dans la direction de chargement (14), (Karaj et al., 2010). 


$$
R_{D R}=\frac{D_{R \max }}{d}
$$

Où $d$ représente soit la longueur, la largeur ou l'épaisseur de la graine (mm).

La dureté H (N/mm) est selon Karaj et al., (2010), le rapport entre la charge de rupture $\mathrm{F}_{\mathrm{Rmax}}(N)$ et la déformation au point de rupture $\mathrm{D}_{\mathrm{Rmax}}(\mathrm{mm})$.

$$
H=\frac{F_{R \max }}{D_{R \max }}
$$

L'énergie de rupture $\mathrm{E}_{\mathrm{R}}(\mathrm{N} / \mathrm{mm})$ est l'énergie nécessaire pour rompre l'échantillon (Figure 4). Elle a été déterminée entre le point initial et le point de rupture.



Figure 3: Essai de compression : Charge en fonction de la déformation

\section{Détermination des propriétés mécaniques des amandes de Jatropha}

Cette mesure a permis la détermination de l'énergie nécessaire pour la production d'huile à partir des amandes des graines de Jatropha. On a ainsi pu déduire la relation entre la force de pression utilisée, la déformation relative et les spécifications de l'amande. Les essais d'extraction d'huile (séparation solide-liquide) ont été effectués sur 4 échantillons à la température ambiante $\left(23-24^{\circ} \mathrm{C}\right)$.

\section{Force de compression}

La vitesse de compression de la machine d'extraction est de $40 \mathrm{~mm} / \mathrm{mn}$, avec comme charges de consigne en compression respectives de $1000 \mathrm{~N}, 5000$ $\mathrm{N}$ et $12000 \mathrm{~N}$. Chaque graine est pressée dans une position plate (suivant l'épaisseur), (Figure 5) favorisant une grande surface de pressage (Vursavus et Ozguven, 2004 ; Hasheminia, 2007). Selon (Murthy et al., 1998) et (Tavakoli et al., 2009), une telle simplification de la surface des amandes s'est avérée suffisante pour déterminer la contrainte de compression des graines oléagineuses. 
A partir des données réelles des essais, l'application Curve Fitting intégrée dans MATLAB, établie la relation mathématique entre la force de compression et la déformation des graines (équation 16)

$F(\Delta l)=A \cdot \exp (-B . \Delta l)+C$

$F(N)$ est l'intensité de la force de compression ; $\Delta l(\mathrm{~mm})$ la déformation des amandes et $A(N), B(1 / \mathrm{mm})$ et $C(N)$ les constantes à déterminer



Figure 4: Dispositif expérimental pour les essais de compression des amandes de graines de Jatropha (a) 1-support ; 2-bras du piston ; 3-amande de graine de Jatropha (b) Dispositif réel

\section{Contrainte de compression :}

La déformation relative s'obtient par l'équation (17)

$\varepsilon=\frac{\Delta l}{l} \Leftrightarrow \Delta l=\varepsilon \cdot l$

En modifiant l'équation (17) et en divisant l'équation (16) par la surface projetée $\mathbf{S}(\mathrm{mm})$, l'on a obtenu l'expression de la contrainte de compression :

$\sigma(\varepsilon)=\frac{F(l \cdot \varepsilon)}{S}=\frac{A \cdot \exp (-B \cdot l \cdot \varepsilon)+C}{S}$

Trois coefficients ont été introduits : $\mathrm{D}(M P a), \mathrm{F}(-)$, et $\mathrm{G}(M P a)$

Ce qui permet de réduire l'expression de la contrainte de compression $\sigma(M P a)$ par :

$\sigma(\varepsilon)=D \cdot \exp (-F \cdot \varepsilon)+G$

Energie :

L'énergie volumique $\mathrm{W}\left(\mathrm{J} / \mathrm{mm}^{3}\right)$ est calculée en intégrant la fonction de contraintes (équation (19)) qui a donné la quantité d'énergie nécessaire pour déformer une unité de volume.

$W(\varepsilon)=\int_{\varepsilon_{1}}^{\varepsilon_{2}} \sigma(\varepsilon) d \varepsilon=\int_{\varepsilon_{1}}^{\varepsilon_{2}}(D \cdot \exp (-F \cdot \varepsilon)+G) d \varepsilon$

En effectuant le calcul intégral de l'équation (21), l'on a obtenu l'expression de l'énergie nécessaire pour extraire l'huile de l'amande des graines de Jatropha. 


$$
W(\varepsilon)=\left[\frac{D}{F} \exp (-F \cdot \varepsilon)+G \varepsilon+H\right]_{\varepsilon_{1}}^{\varepsilon_{2}}
$$

Evaluation de la qualité de l'ajustement des modèles : Analyse statistique

Le tracé des résidus et les limites de prédiction sont des méthodes graphiques qui facilitent l'interprétation visuelle, tandis que le calcul statistique et les limites de confiance des coefficients donnent des mesures numériques qui facilitent le raisonnement statistique.

\section{Carré des résidus (Sum of Square Errors (SSE))}

Cette donnée statistique mesure l'écart total entre les valeurs de l'ajustement et les données réelles. Elle est également appelée carré des résidus, généralement noté $S S E$.

$$
S S E=\sum_{i=1}^{n} w_{i} \cdot\left(y_{i}-f_{i}\right)^{2}
$$

Où $y_{i}$ est la valeur des données réelles, $f_{i}$ la valeur de l'ajustement et $w_{i}$ le coefficient de pondération appliqué à chaque point, généralement l'on prend $w_{i}=1$.

\section{Coefficient de détermination ( $R$-square)}

Le coefficient de détermination $\mathrm{R}^{2}$, est un indicateur qui permet de juger la qualité d'une régression.

$$
R^{2}=1-\frac{\sum_{i=1}^{n} w_{i} \cdot\left(y_{i}-f_{i}\right)^{2}}{\sum_{i=1}^{n} w_{i} \cdot\left(y_{i}-y_{\text {moy }}\right)^{2}}=1-\frac{S S E}{S S T}
$$

Où $y_{\text {moy }}$ est la moyenne des données observées et SST est la somme totale des carrés.

Ce coefficient varie entre 0 et 1 , donnant ainsi un pouvoir de prédiction faible et un pouvoir de prédiction fort.

\section{Coefficient de détermination ajusté (Adjusted R-square)}

Cette donnée statistique utilise le coefficient de détermination $\mathrm{R}^{2}$ définie ci-dessus et l'ajuste en fonction des degrés de liberté résiduels.

$$
v=n-m
$$

$v$ indique le nombre d'informations indépendantes impliquant les $n$ points de données nécessaires au calcul de la somme des carrés.

$$
R_{\text {ajusté }}^{2}=1-\frac{S S E(n-1)}{S S T(v)}
$$

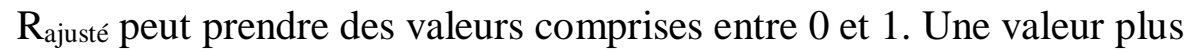
proche de 1 indique un meilleur ajustement. 


\section{Erreur quadratique moyenne (Root Mean Square Error (RMSE))}

L'erreur quadratique moyenne est une estimation de l'écart type de la composante aléatoire dans les données et elle est définie par :

$R M S E=s=\sqrt{(M S E)}=(M S E)^{1 / 2}$

Où $M S E$ est l'erreur quadratique moyenne ou le carré moyen résiduel : $M S E=$ SSE

\section{Résultats et discussion}

Propriétés physiques des graines et amandes de Jatropha curcas :

\section{Caractéristiques dimensionnelles des graines et amandes de Jatropha}

Les valeurs moyennes et l'écarts-type des dimensions des graines et amandes de Jatropha, pour un échantillon de 100 graines, sont présentées dans le tableau 1.

Tableau 1: Dimensions moyennes des graines et amandes de Jatropha.

\begin{tabular}{llll}
\hline & Longueur $(\mathbf{m m})$ & Largeur $(\mathbf{m m})$ & Epaisseur $(\mathbf{m m})$ \\
\hline Graine & $18,06 \pm 0,75$ & $11,34 \pm 0,72$ & $8,29 \pm 0,78$ \\
Amande & $14,04 \pm 0,94$ & $8,01 \pm 0,84$ & $6,52 \pm 0,88$ \\
\hline
\end{tabular}

Les tailles des graines et d'amandes de Jatropha illustrées sur les Figures 6,7 et 8 présentent une disparité remarquable de par les différentes mesures effectuées sur l'échantillon de 100 graines.

Pour ce qui est de la longueur, elle est de l'ordre de 17 à $19 \mathrm{~mm}$ pour les graines et de 13 à $15 \mathrm{~mm}$ pour les amandes (Figure 6). Ceci donne un taux de distribution de $78 \%$ pour les graines tandis que les amandes ne sont qu'à $66 \%$.
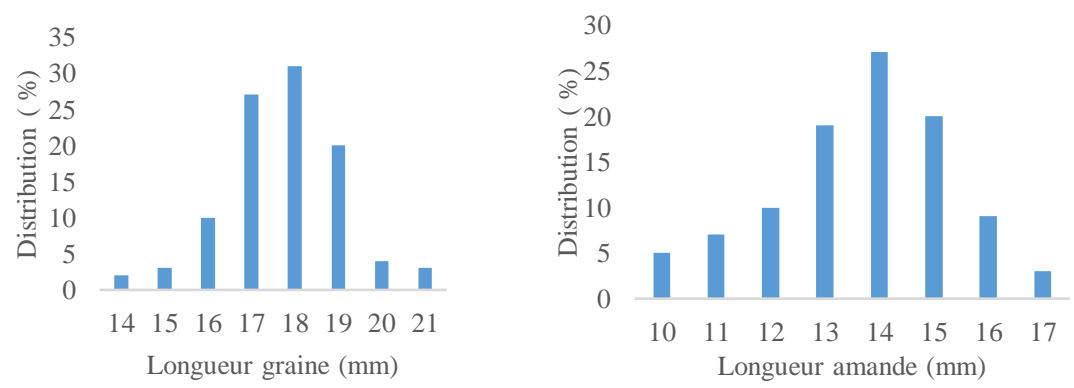

Figure 5: Distribution des longueurs (L) des graines et des amandes de Jatropha

La largeur quant à elle est comprise entre 10 et $12 \mathrm{~mm}$ pour les graines pendant que celle des amandes est comprise entre 7 et $9 \mathrm{~mm}$ (Figure 7). Le taux de distribution dans ce cas est alors respectivement de $68 \%$ et $73 \%$ pour les graines et les amandes. 

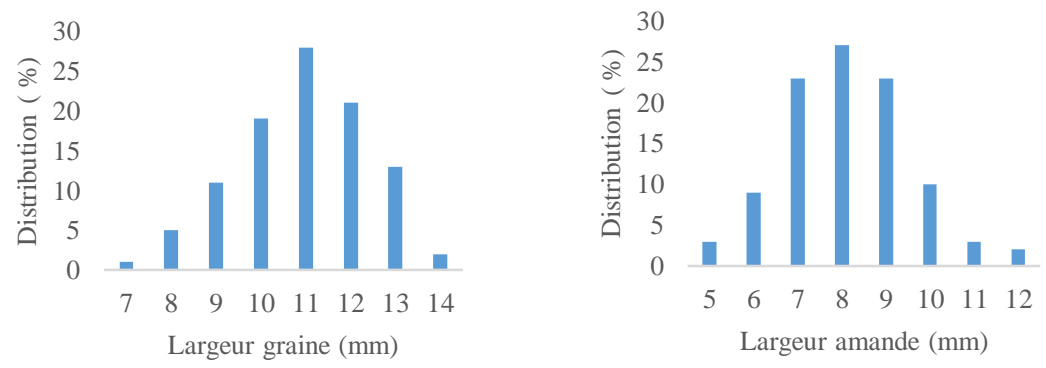

Figure 6: Distribution des largeurs (1) des graines et des amandes de Jatropha

L'épaisseur est comprise entre 7 et $9 \mathrm{~mm}$ pour les graines alors que celle des amandes est comprise entre 5 et $7 \mathrm{~mm}$ (Figure 8). La distribution est de ce fait de l'ordre de $78 \%$ pour les graines et de $79 \%$ pour les amandes.
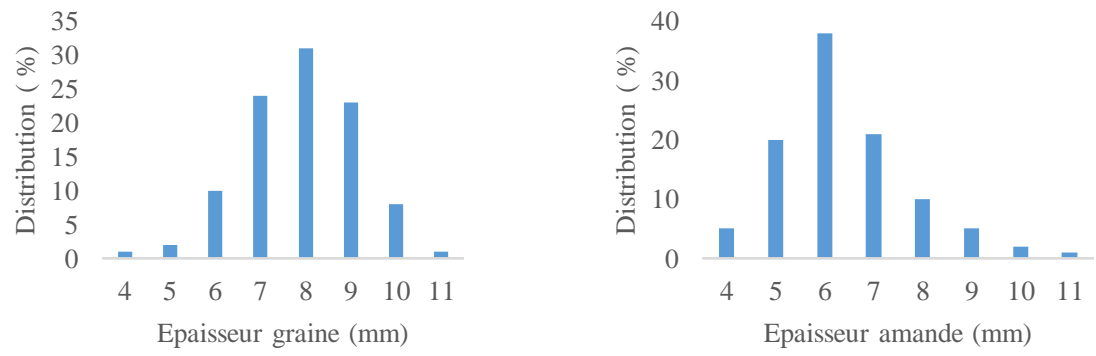

Figure 7: Distribution des épaisseurs (e) des graines et des amandes de Jatropha

Les dimensions des graines telles que la longueur, la largeur et l'épaisseur sont respectivement de $18,06 \pm 0,75 \mathrm{~mm}, 11,34 \pm 0,72 \mathrm{~mm}$ et $8,29 \pm 0,78$ $\mathrm{mm}$ (Tableau 1). Celles des amandes donnent respectivement 14,04 $\pm 0,94$ $\mathrm{mm}, 8,01 \pm 0,84 \mathrm{~mm}$ et $6,52 \pm 0,88 \mathrm{~mm}$. Une différence significative a été observée entre les graines provenant de l'Extrême-Nord Cameroun et celles de Kaffrine au Sénégal. Ainsi les dimensions des amandes et des graines des essences des zones humides ou subhumides ont été supérieures à celles de zones arides. Ces résultats sont semblables à ceux obtenus par Ghosh et Singh (2011) en utilisant les graines provenant de six zones de l'Inde. Les travaux de Ly et al. (2015) sur des graines provenant de deux zones du Sénégal ont également fourni les mêmes résultats.

\section{Caractéristiques massiques et volumiques des graines et amandes de Jatropha curcas $L$.}

Les valeurs des différentes mesures expérimentales sont consignées dans le tableau 2. Les teneurs en eau en base sèche des graines et amandes sont respectivement de 7,69\% et 6,47\%. Les masses moyennes de 1000 graines de $J$. curcas sont de $660 \pm 0,72 \mathrm{~g}$ contre $510 \pm 0,37 \mathrm{~g}$ d'amandes soit $(77,27 \%$ 
$\pm 0,52)$. Un kilogramme de graine occupe une surface de $1,35 \mathrm{~m}^{2}$ contre 0,81 $\mathrm{m}^{2}$ pour les amandes.

Tableau 2: Désignation des caractéristiques massiques des graines et amandes de Jatropha curcas $L$.

\begin{tabular}{lll} 
Désignation & Valeurs & \\
\cline { 2 - 3 } & Graines & Amandes \\
\hline Teneur en eau (base sèche) $(\%)$ & 7,92 & 6,47 \\
Masse de 1000 graines $(\mathrm{g})$ & $660 \pm 0,72$ & $510 \pm 0,37$ \\
Aire massique $\left(\mathrm{m}^{2} / \mathrm{kg}\right)$ & $1,35 \pm 0,01$ & $0,81 \pm 0,02$ \\
Pourcentage poids amande/ poids graine $(\%)$ & $77,27 \pm 0,52$ & \\
Pourcentage poids coques/ poids graine $(\%)$ & $22,73 \pm 0,48$ & \\
\hline
\end{tabular}

Le tableau 3 regroupe les paramètres volumiques des graines et amandes. Le volume d'une graine de Jatropha est de $1,7 \mathrm{~cm}^{3}$; il est environ 2 fois supérieur à celui de son amande. La masse volumique des amandes est 1,5 fois celle des graines. La masse volumique apparente présente des ratios respectifs $0,69 \%$ et $0,71 \%$ des graines et amandes de Jatropha

Tableau 3: Caractéristiques volumiques des graines et amandes de Jatropha curcas $L$.

\begin{tabular}{lll}
\hline \multirow{2}{*}{ Désignation } & Valeurs & \\
\cline { 2 - 3 } & Graines & Amandes \\
\hline Teneur en eau (base sèche), $(\%)$ & 7,920 & 6,470 \\
Volume de 1000 graines $\left(\mathrm{cm}^{3}\right)$ & $1701,230 \pm 67,000$ & $723,480 \pm 710$ \\
Masse volumique réelle $\left(\mathrm{kg} / \mathrm{m}^{3}\right)$ & $294,120 \pm 0,370$ & $684,310 \pm 0,420$ \\
Masse volumique apparente $\left(\mathrm{kg} / \mathrm{m}^{3}\right)$ & $202,94 \pm 0,26$ & $485,860 \pm 0,310$ \\
Volume massique $\left(\mathrm{m}^{3} / \mathrm{kg}\right)$ & $0,020 \pm 0,00003$ & $0,0015 \pm 0,0000009$ \\
Ratio masse volumique & $0,69 \pm 0,0018$ & $0,71 \pm 0,0009$ \\
Porosité $(\%)$ & $44,93 \pm 4,2$ & $40,84 \pm 1,4$ \\
\hline
\end{tabular}

\section{Paramètres de forme des graines et amandes de Jatropha curcas $\boldsymbol{L}$.}

Le tableau 4 présente les valeurs obtenues pour les paramètres morphométriques des graines et amandes de Jatropha La largeur est 1,37 fois l'épaisseur de la graine et la longueur de l'amande est 1,23 fois celle de l'épaisseur (Degré d'aplatissement). La valeur de l'élongation indique que la longueur de la graine est 1,59 fois supérieure à sa largeur contre 1,75 fois pour l'amande. Le diamètre équivalent ou le diamètre de la sphère qui aurait le même volume que la graine de Jatropha est environ 14,81 mm et 11,13 pour l'amande. Le diamètre équivalent de la graine est 1,33 fois supérieur à celui de l'amande. Les diamètres basés sur les moyennes arithmétiques (12,57 \pm $0,75 \mathrm{~mm}$ et $9,52 \pm 0,89 \mathrm{~mm})$ et géométriques $(11,94 \pm 0,72 \mathrm{~mm}$ et 9,01 \pm $0,85 \mathrm{~mm}$ ) sont relativement proches de leur diamètre équivalent à $3 \mathrm{~mm}$ près. Ces résultats sont similaires à ceux de Garnayak et al. (2008). 
Tableau 4 : Désignation des paramètres de forme des graines et amandes de Jatropha curcas $L$.

\begin{tabular}{lll} 
Désignation & \multicolumn{2}{l}{ Valeurs } \\
\cline { 2 - 3 } & Graines & Amandes \\
\hline Degré d'aplatissement & $1,37 \pm 0,97$ & $1,23 \pm 0,98$ \\
Elongation & $1,59 \pm 0,77$ & $1,75 \pm 0,75$ \\
Sphéricité & $0,66 \pm 0,04$ & $0,64 \pm 0,11$ \\
Diamètre équivalent $(\mathrm{mm})$ & $14,81 \pm 0,92$ & $11,13 \pm 0,79$ \\
Moyenne arithmétique du diamètre $(\mathrm{mm})$ & $12,57 \pm 0,75$ & $9,52 \pm 0,89$ \\
Moyenne géométrique du diamètre $(\mathrm{mm})$ & $11,94 \pm 0,72$ & $9,01 \pm 0,85$ \\
\hline
\end{tabular}

La sphéricité des graines d'une valeur de 0,66 est identique à celle obtenue par Garnayak et al. (2008). Alors que les travaux de Sirisomboon et $a l$. (2007) ont rapporté des valeurs de sphéricité plus élevées pour les graines que pour les amandes. Elles ont été de 0,64 pour les graines et 0,68 pour les amandes.

\section{Propriétés mécaniques des graines de Jatropha curcas}

\section{Etude de compression à grande vitesse $(40,20,10$ et $5 \mathrm{~mm} / \mathrm{mn})$}

Pour les grandes vitesses telles que $5 ; 10 ; 20$ et $40 \mathrm{~mm} / \mathrm{mn}$, les essais de compression sont très brefs (en moyenne 2 à 5 secondes). Les courbes des différents essais de compression à grande vitesse (5 à $40 \mathrm{~mm} / \mathrm{mn})$ donnent une allure identique à celle obtenue par Karaj et al., 2010. Les courbes d'essai à ces différentes vitesses présentent des ruptures de la coque en plusieurs points (figure 9a).
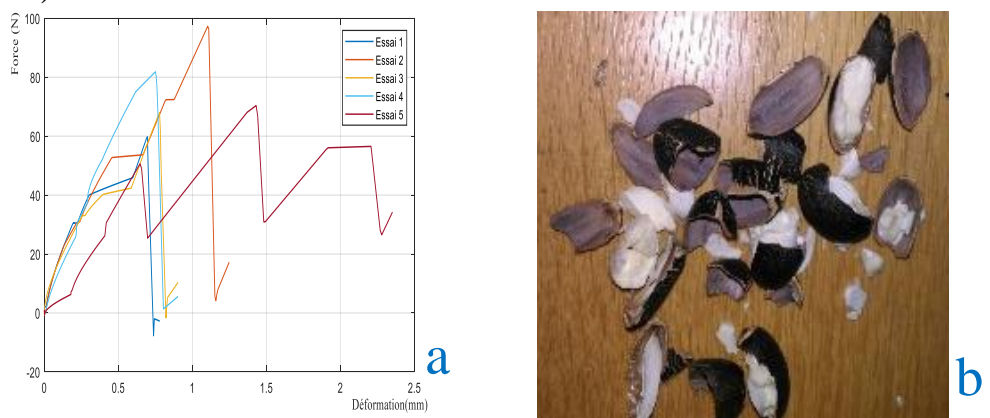

Figure 8: (a) Essai de Compression des graines de Jatropha pour des vitesses à grande vitesse (b) Brisure par Compression des graines de Jatropha

Le Tableau 5 présente les propriétés mécaniques des graines de Jatropha obtenues lors des essais de compression à grande vitesse. Ce sont la charge de rupture $F_{R \max }(N)$, la déformation au point de rupture $D_{R \max }(\mathrm{mm})$, le taux de déformation au point de rupture $R_{D R}$, et la dureté $H(\mathrm{~N} / \mathrm{mm})$. 
Tableau 5 : Propriétés mécaniques des graines de Jatropha curcas $L$. en fonction de la vitesse de compression

\begin{tabular}{llllllllll}
\hline Essai & $\begin{array}{l}\text { Vitesse } \\
(\mathrm{mm} / \mathrm{mn})\end{array}$ & $\begin{array}{l}\text { Temps } \\
(\mathrm{s})\end{array}$ & $\begin{array}{l}\text { Limite } \\
(\mathrm{mm})\end{array}$ & $F_{R \max }$ & $D_{R \max }$ & $F_{R}$ & $D_{R}$ & $\boldsymbol{R}_{D R}$ & $H$ \\
\hline $\mathbf{1}$ & 40 & $01: 16$ & 7,51 & 97,98 & 1,11 & 17,53 & 1,25 & 0,15 & 88,31 \\
$\mathbf{2}$ & 40 & $01: 15$ & 7,51 & 46,27 & 2,25 & 12,69 & 2,28 & 0,30 & 20,60 \\
$\mathbf{3}$ & 40 & $01: 14$ & 7,51 & 84,54 & 1,06 & 38,91 & 2,21 & 0,14 & 80,09 \\
$\mathbf{4}$ & 40 & $01: 14$ & 7,51 & 82,01 & 0,75 & 5,80 & 0,91 & 0,10 & 108,90 \\
$\mathbf{5}$ & 40 & $01: 13$ & 7,50 & 70,61 & 1,44 & 34,71 & 2,36 & 0,19 & 49,17 \\
& & & & & & & & & \\
$\mathbf{1}$ & 20 & $01: 12$ & 7,52 & 158,82 & 2,01 & 42,56 & 2,05 & 0,27 & 78,91 \\
$\mathbf{2}$ & 20 & $01: 11$ & 7,50 & 133,81 & 1,63 & 13,35 & 1,68 & 0,22 & 82,29 \\
$\mathbf{3}$ & 20 & $01: 10$ & 7,51 & 83,13 & 1,27 & 7,63 & 1,33 & 0,17 & 65,29 \\
$\mathbf{4}$ & 20 & $01: 09$ & 7,51 & 33,02 & 0,91 & 20,63 & 1,43 & 0,12 & 36,30 \\
$\mathbf{5}$ & 20 & $01: 08$ & 7,50 & 115,78 & 1,91 & 57,49 & 2,66 & 0,25 & 60,77 \\
& & & & & & & & & \\
$\mathbf{1}$ & 10 & $01: 07$ & 7,50 & 50,17 & 0,94 & 14,57 & 1,32 & 0,13 & 53,16 \\
$\mathbf{2}$ & 10 & $01: 06$ & 7,52 & 85,99 & 1,27 & 56,58 & 2,06 & 0,17 & 67,78 \\
$\mathbf{3}$ & 10 & $01: 04$ & 7,50 & 78,81 & 1,00 & 40,41 & 1,52 & 0,13 & 78,66 \\
$\mathbf{4}$ & 10 & $01: 03$ & 7,55 & 59,05 & 0,91 & 40,02 & 2,15 & 0,12 & 65,22 \\
$\mathbf{5}$ & 10 & $01: 02$ & 7,50 & 137,38 & 1,56 & 47,87 & 1,59 & 0,21 & 87,87 \\
& & & & & & & & & \\
$\mathbf{1}$ & 5 & $00: 37$ & 7,57 & 146,23 & 1,90 & 44,80 & 1,93 & 0,25 & 76,82 \\
$\mathbf{2}$ & 5 & $00: 35$ & 7,50 & 126,96 & 1,45 & 23,87 & 1,46 & 0,19 & 87,38 \\
$\mathbf{3}$ & 5 & $00: 33$ & 7,61 & 56,82 & 0,78 & 45,73 & 1,85 & 0,10 & 73,13 \\
$\mathbf{4}$ & 5 & $00: 32$ & 7,50 & 125,95 & 2,12 & 33,27 & 2,14 & 0,28 & 59,33 \\
$\mathbf{5}$ & 5 & $00: 30$ & 7,50 & 157,34 & 1,77 & 32,43 & 1,78 & 0,24 & 89,03 \\
\hline
\end{tabular}

Les essais de compression effectués sur les graines de Jatropha collectées dans la Région de l'Extrême-Nord Cameroun avec une vitesse de déformation comprise entre 5 et $40 \mathrm{~mm} / \mathrm{mn}$ ont permis d'obtenir des résultats suivants : la déformation au point de rupture $D_{R \max }=2,35 \pm 0,67 \mathrm{~mm}$; le taux de déformation au point de rupture $R_{D R}=0,20 \pm 0,07$; la dureté $H=69,41 \pm 5,32$ $\mathrm{N} / \mathrm{mm}$ à l'exception de la charge de rupture $F_{R \max }(N)$ qui présente une différence significative soit de l'ordre de $52 \%$. Ces essais à grande vitesse sont rapides en moyenne $1 \mathrm{mn} 15$ secondes et les résultats obtenus présentent des brisures d'amandes (figure $9 \mathrm{~b}$ ) ; ce qui engendrera un coût considérable sur le processus de vannage. Ainsi, la détermination du module d'élasticité en compression est importante pour spécifier correctement la rigidité et la dureté des graines tel qu'observé par Hasheminia, (2007). Les essais à faible vitesse 
permettent d'avoir un comportement linaire des propriétés caractéristiques et structurelles de la graine. Ces résultats concordent avec ceux de Sirisomboon et al., (2007). En effet, leurs travaux ont montré une dureté de 69,98 N/mm pour les graines avec une force de rupture de $146,63 \mathrm{~N}$.

\section{Etude de compression à vitesse réduite $(1 \mathrm{~mm} / \mathrm{mn})$}

La courbe des différents essais de compression à $1 \mathrm{~mm} / \mathrm{mn}$ (Figure 10a) a une allure identique à la loi de comportement conventionnelle existant. Une zone de déformation réversible a été générée et la courbe a été parfaitement linéaire. Elle a un comportement Hookien et un palier d'écoulement a permis de déterminer la zone des déformations irréversibles. Le décrochage des dislocations (glissement atomique) a déterminé une limite d'élasticité haute et une limite d'élasticité basse. Le tableau 6 présente les valeurs des propriétés structurelles des graines de Jatropha effectué sur cinq (5) échantillons de graine. La charge de rupture $F_{R \max }(N)$ nécessaire pour briser la coque des graines est comprise entre 143,05 et 146,69N. Ces résultats ont été confirmés par ceux obtenus par Sirisomboon et al. (2007) et Karaj et al. (2010).

Tableau 6 : Propriétés structurelles de la graine de Jatropha curcas $L$.

\begin{tabular}{cccccc}
\hline Essai & $F_{e, h}(\mathrm{~N})$ & $F_{e, b}(\mathrm{~N})$ & $F_{e}(\mathrm{~N})$ & $F_{r}(\mathrm{~N})$ & $K=E S / L(\mathrm{~N} / \mathrm{mm})$ \\
\hline 1 & 78 & 74 & 74 & 143,05 & 100 \\
3 & 110 & 100 & 100 & 119,18 & 100 \\
4 & 114 & 110 & 110 & 146,26 & 120 \\
5 & 110 & 100 & 100 & 129,15 & 100 \\
\hline
\end{tabular}

La charge de rupture $\mathrm{F}_{\text {Rmax }}$ moyenne, nécessaire pour briser la coque des graines de Jatropha est de 134,41 N pour une raideur moyenne de $\mathrm{K}=105$ $\mathrm{N} / \mathrm{mm}$; avec cette valeur de la raideur, la rupture de la coque est parfaite et l'amande de la graine de Jatropha est extraite entièrement (Figure 10b).
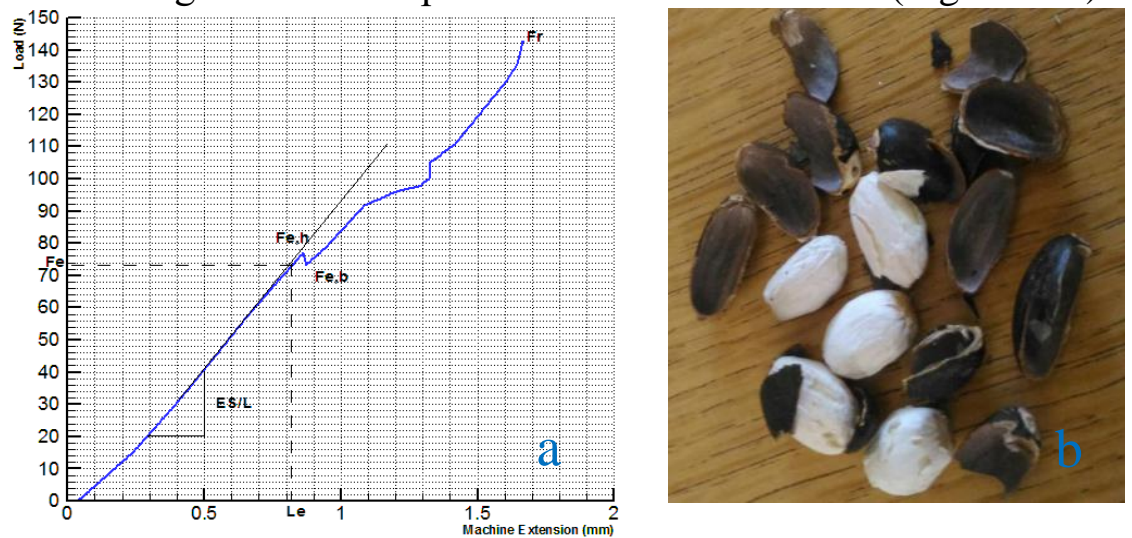

Figure 9: Courbes des essais de compression des graines de Jatropha à la vitesse de 1 $\mathrm{mm} / \mathrm{min}$. (a) Rupture de la coque de la graine de Jatropha (b) 


\section{Détermination des propriétés mécaniques des amandes des graines de Jatropha \\ Force de Pression}

Le Tableau 7 donne la hauteur et la surface moyenne de chaque graine avant les tests de compression. Les tests de compression ont été effectués avec des charges de 1000 N, 5000 N et 12000 N. Les courbes de régression des différents essais et leurs modèles mathématiques ont été obtenus en utilisant l'algorithme de Trust-Region (Figure 11).

Tableau 7 : Propriétés physique des amandes de graines de Jatropha curcas $L$.

Propriétés Essai d'extraction d'huile de graine Jatropha curcas $L$.

\begin{tabular}{|c|c|c|c|c|}
\hline & 1 & 2 & 3 & 4 \\
\hline eur moyenne $(\mathrm{mm})$ & $7,50 \pm 0,85$ & $7,51 \pm 0,84$ & $7,51 \pm 0,57$ & $7,51 \pm 1,02$ \\
\hline $\begin{array}{l}\text { Irface projetée moyenne } \\
\left.m^{2}\right)\end{array}$ & $112,46 \pm 10,35$ & $131,92 \pm 11,21$ & $132,57 \pm 7,43$ & $111,92 \pm 11,52$ \\
\hline
\end{tabular}


Figure 10: Approximation de la force de compression en fonction de la déformation des amandes de Jatropha (a) film d'huile de Jatropha lors de l'essai de compression des amandes

(b)

La régression a été obtenue avec un coefficient de détermination de 0,99. Le tableau 8 présente les coefficients obtenus avec 95\% d'intervalle de confiance.

Tableau 8 : Analyse statistique de la modélisation de la force de compression des amandes de Jatropha.

\begin{tabular}{|c|c|c|c|c|c|c|c|c|c|c|}
\hline $\mathrm{N}^{\circ}$ & $\begin{array}{l}\mathrm{F}_{\mathrm{C}} \\
(\mathrm{N})\end{array}$ & $\begin{array}{l}\mathrm{A} \\
(\mathrm{N})\end{array}$ & $\begin{array}{l}\text { B } \\
(1 / \mathrm{mm})\end{array}$ & $\begin{array}{l}\mathrm{C} \\
(\mathrm{N})\end{array}$ & Coeff. & DFE & SSE & RMSE & $\mathrm{R}^{2}$ & $\mathrm{R}^{2}$ ajusté \\
\hline 1 & 994,60 & 14,32 & $-0,78$ & $-15,84$ & 3 & 293 & 3120,28 & 3,26 & 0,99 & 0,99 \\
\hline 2 & 5000,60 & 4,98 & $-0,95$ & $-5,83$ & 3 & 293 & 5393,97 & 4,29 & 0,99 & 0,99 \\
\hline 3 & 5009,40 & 3,62 & $-1,03$ & $-2,32$ & 3 & 293 & 10384,97 & 5,95 & 0,99 & 0,99 \\
\hline 4 & 11142,00 & 12,71 & $-0,98$ & $-13,66$ & 3 & 293 & 5077,64 & 13,16 & 0,99 & 0,99 \\
\hline
\end{tabular}


Le modèle mathématique de la force de compression pour une extraction de l'huile de Jatropha a été obtenu à partir de la moyenne des quatre essais :

$$
\mathrm{F}(\Delta \mathrm{l})=8,91 \cdot \operatorname{Exp}(0.94 \cdot \Delta \mathrm{l})-9,41(\mathrm{~N})
$$

\section{Contrainte de compression et module d'élasticité}

Les caractéristiques des amandes de Jatropha du tableau 7 et les données des essais de compression ont permis de déterminer l'approximation mathématique de la contrainte de compression obtenu en utilisant l'algorithme de Levenberg-Marquardt intégré dans l'application MATLAB. La courbe d'approximation de compression et le modèle obtenu pour chaque essai est présentée sur la figure 12 .

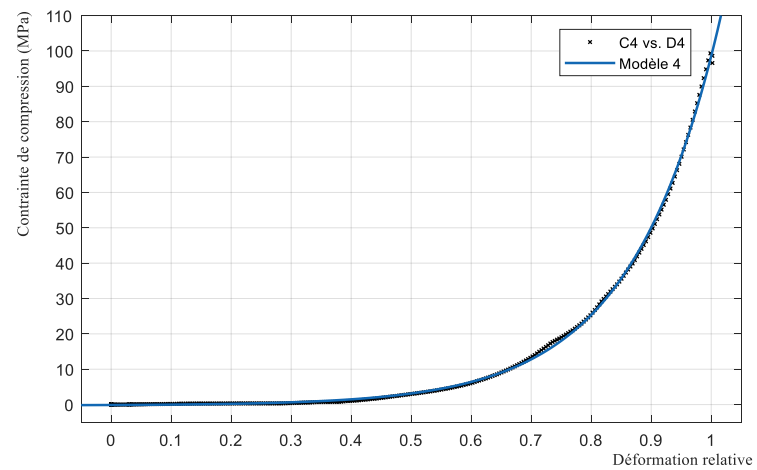

Figure 11: Contraintes de compression en fonction de la déformation relative des amandes de graine de Jatropha.

La régression a été obtenue avec un coefficient de détermination de 0,99. Le tableau 9 présente les coefficients obtenus avec 95\% d'intervalle de confiance.

Tableau 9: Analyse statistique de la modélisation des contraintes de compression des amandes de Jatropha

\begin{tabular}{llllllllll}
\hline $\mathrm{N}^{\circ}$ & $\begin{array}{l}\mathrm{D} \\
(\mathrm{MPa})\end{array}$ & $\mathrm{F} \mathrm{(-)}$ & $\begin{array}{l}\mathrm{G} \\
(\mathrm{MPa})\end{array}$ & Coeff. & DFE & SSE & RMSE & $\mathrm{R}^{2}$ & $\mathrm{R}^{2}$ ajusté \\
\hline 1 & 0,13 & $-5,83$ & $-0,14$ & 3 & 293 & 0,25 & 0,03 & 0,99 & 0,99 \\
2 & 0,04 & $-7,11$ & $-0,05$ & 3 & 293 & 0,31 & 0,03 & 0,99 & 0,99 \\
3 & 0,03 & $-7,76$ & $-0,02$ & 3 & 293 & 0,59 & 0,05 & 0,99 & 0,99 \\
4 & 0,11 & $-6,83$ & $-0,12$ & 3 & 293 & 3,22 & 0,10 & 0,99 & 0,99 \\
\hline
\end{tabular}

Le modèle mathématique de la contrainte de compression pour l'extraction de l'huile de Jatropha a été obtenu en utilisant la moyenne des quatre essais.

$$
\sigma(\varepsilon)=0,08 \cdot \operatorname{Exp}(6,89 \cdot \varepsilon)-0,08(\mathrm{MPa})
$$

Les courbes des contraintes de compression $\sigma(\mathrm{N})$ en fonction de la déformation relative $\varepsilon(-)$ présentent toute une description plus détaillée du 
processus d'extraction de l'huile de Jatropha Selon Herak et al., (2010). Chaque courbe a indiqué clairement qu'elle peut être segmentée en trois phases distinctes (Figure 13). Dans la phase I, le module d'élasticité en compression reste constant et la courbe de la contrainte en fonction de la déformation relative reste linéaire, analogue à la loi de Hooke. Théoriquement, il n'y a pas de déformation permanente pendant la phase d'extraction de l'huile. La déformation relative augmente et se rapproche de la première limite d'extraction $\varepsilon_{\text {I. }}$. La phase d'extraction commence et se mélange à la phase II. Dans cette phase, la plus grande quantité d'huile est extraite et le module d'élasticité n'est plus constant. Il suit une progression non linéaire. La phase finale quant à elle nécessite beaucoup d'énergie, le module d'élasticité peut être considéré comme une constante.

Les phases I et III peuvent donc être considérées comme linéaires. Les différentes courbes peuvent être remplacées par des lignes droites. Dans la phase I, la ligne droite passe par deux extrémités, le point zéro qui correspond à une ordonnée nulle et le point limite $\varepsilon_{\mathrm{I}}$ qui correspond au point huileux inferieur. Ce dernier est déterminé en utilisant l'algorithme de LevenbergMarquardt et vérifié par la recherche d'un coefficient de détermination minimum par rapport à l'initiale à l'aide du logiciel MATLAB R2018a. La différence entre la contrainte calculée avec la courbe et celle calculée en utilisant la ligne droite est pratiquement identique pour tous les essais effectués soit $\Delta \sigma \cong 0,009 \mathrm{MPa}$. On peut s'attendre à ce que la ligne droite dans la phase III soit déterminée avec les mêmes précisions. Le point $\varepsilon_{\text {III }}$ (point huileux supérieure) à travers lequel la droite de la phase III passe est déterminé de manière similaire au point limite de la phase I. Les limites entre les différentes phases pour tous les essais sont ainsi présentées dans le Tableau 11.

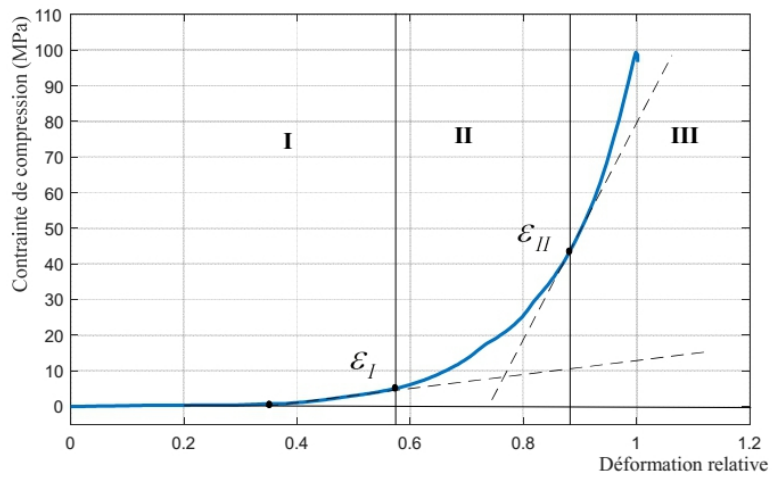

Figure 12: Relation générale, contrainte de compression et déformation relative dans l'amande de Jatropha 


\section{Energie minimum nécessaire pour extraire l'huile de l'amande de Jatropha}

La Figure 14 présente les courbes de volume énergétique de déformation pour le pressage des amandes de graines de Jatropha. Elles sont basées sur l'équation 29 , ce qui détermine :

- L'énergie $W_{I}$ à l'aide de la limite $\varepsilon=[0,00 ; 0,40]$;

- L'énergie $\mathrm{W}_{\text {II }}$ à l'aide de la limite $\varepsilon=[0,00 ; 0,85]$;

- L'énergie $\Delta \mathrm{W}$ nécessaire pour une extraction compris entre $\varepsilon=[0,85$; $0,90]$

$\Delta \mathrm{W}$ représente une énergie de sécurité pour avoir un maximum d'huile extraite et $\Delta \mathrm{p}=\frac{\Delta \mathrm{W}}{W_{I I}}$ représente le pourcentage d'énergie supplémentaire pour une extraction optimale.

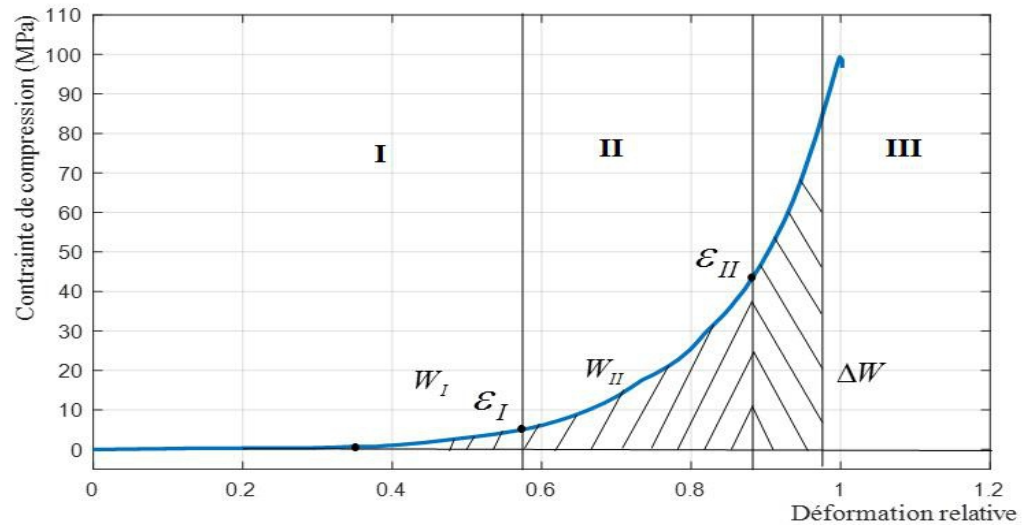

Figure 13: Energie minimum pour extraction à froid d'huile de Jatropha

Les résultats sur la consommation énergétique sont présentés dans le tableau 10. Les essais avec des charges de consignes de $5000 \mathrm{~N}$, donne une extraction d'huile avec une énergie supplémentaire comprise entre 43 et $48 \%$ par contre, à $12000 \mathrm{~N}$, un supplément de $83 \%$ de l'énergie augmenterait le coût d'extraction d'où des charges supplémentaires pour le dimensionnement de la presse à huile de Jatropha

Tableau 10 : Limites des différentes phases lors de l'extraction de l'huile de Jatropha et énergie associée à chaque phase

\begin{tabular}{lllllll}
\hline $\mathrm{N}$ & $\varepsilon_{\mathrm{I}}(-)$ & $\varepsilon_{\mathrm{III}}(-)$ & $\mathrm{W}_{\mathrm{I}}\left(\mathrm{J} / \mathrm{mm}^{3}\right)$ & $\mathrm{W}_{\mathrm{II}}\left(\mathrm{J} / \mathrm{mm}^{3}\right)$ & $\Delta \mathrm{W}\left(\mathrm{J} / \mathrm{mm}^{3}\right)$ & $\Delta \mathrm{p}(\%)$ \\
\hline 1 & - & - & - & - & - & - \\
2 & 0,45 & 0,85 & 0,12 & 2,34 & 1,02 & 43 \\
3 & 0,55 & 0,85 & 0,26 & 2,83 & 1,35 & 48 \\
4 & 0,40 & 0,75 & 0,18 & 2,59 & 2,17 & 83 \\
\hline
\end{tabular}




\section{Paramètres limites de l'extraction d'huile de Jatropha}

Pour une extraction à froid de l'huile d'amandes des graines de Jatropha, la limite des déformations relatives pour une extraction maximale en utilisant une énergie minimum est comprise entre $\varepsilon_{I}=0,40$ (point huileux inferieur) et $\varepsilon_{\text {II }}=0,85$ (point huileux supérieur). Le Tableau 11 illustre les paramètres limites pour une extraction optimale d'huile des amandes.

Tableau 11 : Paramètres limites pour une extraction optimale d'huile d'amande de graines de Jatropha

\begin{tabular}{lllll}
\hline $\begin{array}{l}\text { Déformation } \\
\text { relative } \\
\varepsilon(-)\end{array}$ & $\begin{array}{l}\text { Force de } \\
\text { compression } \\
(N)\end{array}$ & $\begin{array}{l}\text { Contrainte de } \\
\text { compression } \\
(\mathrm{MPa})\end{array}$ & $\begin{array}{l}\text { Module d'élasticité } \\
\text { en compression } \\
(\mathrm{MPa})\end{array}$ & $\begin{array}{l}\text { Energie } \\
\left(\mathrm{J} / \mathrm{mm}^{3}\right)\end{array}$ \\
\hline $0,40 \pm 0,08$ & $72,71 \pm 0,18$ & $0,65 \pm 0,15$ & $4,98 \pm 0,51$ & $0,13 \pm 0,01$ \\
\hline $0,85 \pm 0,06$ & $4905,70 \pm 2,59$ & $37,01 \pm 1,25$ & $12,05 \pm 1,1$ & $2,83 \pm 0,11$ \\
\hline
\end{tabular}

\section{Influence des charges sur l'extraction de l'huile de Jatropha}

Le pressage à chaud d'amandes de graines entraîne une diminution des forces de pression ; ainsi qu'un rendement en huile plus élevé, environ $10 \%$. Par ailleurs, d'autres facteurs peuvent influencer le rendement d'extraction : la technologie de pressage (qui peut être manuelle ou motorisée) et le conditionnement des graines (le tri). Au moment de la récolte, la plante de Jatropha produit $50 \%$ de graines de fruits mûrs qui contiennent une quantité élevée d'huile.

Ce point est important à prendre en compte lors de l'extraction de l'huile, car une température élevée favorise la libération dans l'huile des phospholipides contenus dans les parois cellulaires des graines. Ces derniers sont des facteurs d'encrassement lors d'une utilisation dans des moteurs ou des brûleurs (Vaitilingom, 2007). Une telle dégradation pourrait être évitée en ajoutant un refroidisseur dans la phase intermédiaire du processus de pressage. Cependant, le préchauffage des amandes et le refroidissement nécessite plus d'énergie et donc une augmentation globale de l'énergie consommée.

Pour tous les essais effectués, la courbe de la charge en fonction de la déformation présente une première phase de 0 à 2 secondes, identique pour tous les essais et correspondant à la stabilisation de l'essai pour éviter le jeu et une augmentation de la raideur des graines.

La deuxième phase représente la charge de la machine, la courbe présente un comportement linéaire dans la matière. L'on a des glissements qui se mettent en place à partir de 2,6 à $3 \mathrm{~mm}$ de l'extension de la machine de compression. Il y a des mouvements par rupture des liaisons ou par seuil. Pour les différents essais 1, 2, 3 et 4, l'on observe un seuil de glissement de l'ordre de $0,2 \mathrm{~mm}$ compris entre 2,2 et 3,2 $\mathrm{mm}$ de l'extension de la machine : $\Delta \mathrm{l}_{1}=$ $\Delta \mathrm{l}_{2}=\Delta \mathrm{l}_{3}=\Delta \mathrm{l}_{4}=0,2 \mathrm{~mm}$ (Figure 15). Les temps des différents essais sont respectivement 9,$73 ; 11,65 ; 11,66$ et 12,48 secondes. 


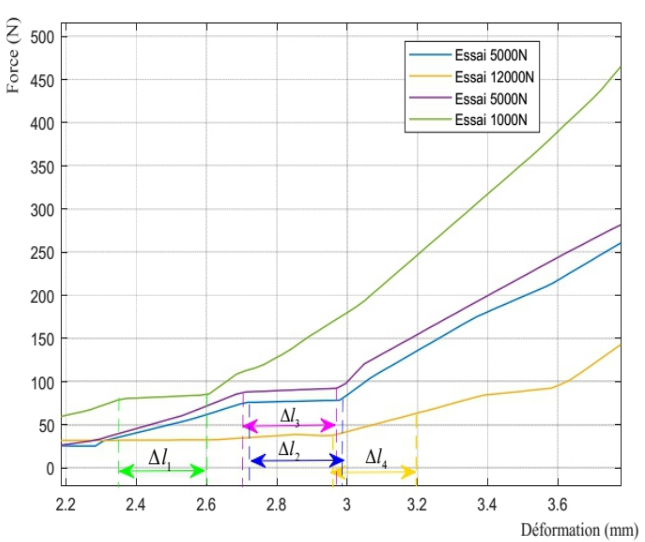

Figure 14: Seuil de rupture et de déplacement interne de molécule.

\section{Conclusion}

Les graines et les amandes de Jatropha curcas ont une forme géométrique proche d'un ellipsoïde ovoïde. Les relations entre longueur, largeur, épaisseur, moyenne arithmétique, moyenne géométrique, volume, sphéricité, masse volumique apparente et réelle, porosité, surface et surface spécifique suivent un modèle bien défini; ce qui permet de fiabiliser la corrélation des dimensions géométriques à la masse unitaire. Ces corrélations pourraient être utilisées pour trier les graines de Jatropha car elles reflètent également les classes de qualité en termes de teneur en huile.

Les propriétés mécaniques telles que la force de rupture, la déformation au point de rupture, le taux de déformation au point de rupture, la dureté ont donné respectivement $143,05 \pm 3,22 \mathrm{~N} ; 2,67 \pm 0,33 \mathrm{~mm} ; 60,47 \pm 4,55 \mathrm{~N} / \mathrm{mm}$ et s'utilisent pour concevoir un système de décorticage. Le fractionnement des graines en fonction de la masse unitaire et des propriétés mécaniques corrélées permet sans doute l'ajustement de manière optimale des équipements de décorticage.

Lors de l'extraction de l'huile des amandes, la détermination des propriétés mécaniques telles que la force et contrainte de compression ont généré respectivement $72,71-4905,7 \mathrm{~N}$ et $0,65-37,01 \mathrm{MPa}$. Cela a permis de déterminer les limites de consommation d'énergie pour une extraction optimale de l'huile des amandes; soit $0,13-2,83 \mathrm{~J} / \mathrm{mm}^{3}$. Il a été observé que les graines et les amandes les plus lourdes sont caractérisées par une teneur en huile élevée. Ces propriétés peuvent être utilisées comme données d'entrée afin d'augmenter l'efficacité des presses à huile de Jatropha.

\section{Remerciements}

Les auteurs remercient le laboratoire de génie mécanique de la faculté d'ingénierie de l'université Vasile Alexandrii de Bacau - Roumanie pour leur contribution et pour leur appui en matériel. 


\section{References:}

1. Amin, M. N., Hossain, M. A., \& Roy K. C. (2004). Effect of moisture content on some physical properties of lentil seeds. Journal of Food Engineering, Vol. 65(1), pp. 83-87.

2. Ballerini, D., \& Alazard-Toux, N. (2006). Les biocarburants : Etat des lieux, perspectives et enjeux du développement. Paris: Éditions Technip. p.348

3. Bagan, H., \& Yamagata, Y. (2012). Landsat analysis of urban growth: How Tokyo became the world's largest megacity during the last 40 years. Remote Sensing of Environment. Vol 127 doi. 10.1016/j.rse.2012.09.011.

4. Bart-Plange, A., \& Baryeh, E. A. (2003). The physical properties of Category B cocoa beans. Journal of Food Engineering, Vol. 60(3), pp.219-227

5. Calisir, S., Ozcan, M., Haciseferogullari, H., \& Yildiz, M. U. (2005). A study on some physico- chemical properties of Turkey okra (Hibiscus esculenta L.) seeds. Journal of Food Engineering, Vol. 68(1), pp. 73-78.

6. Chasseray, P., (1991). Caractéristiques physiques des grains et de leurs dérivés. In : Godon $\mathrm{B}$ Willm $\mathrm{C}$ éditor. Les industries de première transformation des céréales. Techniques et DocumentationsLavoisier, Londres- New York, p. 694.

7. Daudin A., Maury S., \& Vallee C., (2012). Production de biocarburants à partir de la ressource oléagineuse. OCL 2012; Vol 19(1) pp: 29-38. doi: 10.1684/ocl.2012.0433

8. Djeumako, B. Nzie, W. Samon, J. B., Kinfack, R.A. (2015). Conception et dimensionnement d'un équipement de vannage de graines de Jatropha curcas, European Scientific Journal, vol.11(27) ISSN : $1857-7881$

9. Domergue, M., \& Pirot, R. (2008). Jatropha curcas L. Rapport de synthèse bibliographique, CIRAD, p.118

10. Fotseu, W. N., Sidibe, S. S., Blin, J., \& Girard, P. (2011). Adaptation du moteur diesel Rhino à l'utilisation de l'huile de Jatropha curcas comme carburant, Journée scientifique 2IE, Ouagadougoua, p. 5

11. Garnayak, D. K., Pradhan, R. C., Naik, S.N., \& Bhatnagar, N. (2008). Moisture-dependent physical properties of jatropha seed (Jatropha curcas L.), Industrial Crops and Products, Vol 27(1), pp. 123-129, ISSN 0926-6690,

12. Ghosh, L., \& Singh, L. (2011). Variation in seed and seedling characters of Jatropha curcas L. with varying zones and provenances. Tropical Ecology, Vol. 52 (1), pp. 113-122. 
13. Hartlieb, E., \& Gorriz, D. (2004). Case Study "Jatropha Curcas", 60314 Frankfurt, Germany, pp. 24 -32

14. Hasheminia, S. M. (2007). The study of compressive loading on mechanical properties of soyabean kernel. Proceeding of 3rd International conference TAE 2007. Czech University of Life Sciences Prague. pp. 131-134.

15. Herak, D., Gurdil, G., Sedlacek, A., Dajbych, O., \& Simanjuntak, S. (2010). Energy demands for pressing Jatropha curcas L. seeds. Biosystems Engineering, Vol. 106(4), pp. 527-534.

16. Karaj, S., \& Muller J. (2010). Determination of physical, mechanical and chemical properties of seeds and kernel of Jatropha curcas L. Industrial Crops and Products, Vol. 32(2), pp. 129-138.

17. Lourakis, M. I. A. (2005). A brief description of the LevenbergeMarquardt algorithm implemented by Levmar. Heraklion, Institute of Computer Science ICS, Foundation for Research and Technology Hellas FORTH, p6.

18. Mohsenin, N. N. (1986), Physical properties of plant and animal materials: structure, physical characteristics, and mechanical properties, 2nd rev, and updated ed, Gordon and Breach, New York, p891.

19. Murthy, C.T. \& Bhattacharya, S. (1998). Moisture dependant physical and unaxial compression properties of black pepper. Journal of Food Engineering, Vol.37(2), pp.193-205.

20. Orguven F., Kubilay V., (2004). Some physical, mechanical and aerodynamic properties of pine (Pinus pinea). Journal of Food Engineering Vol. 68(2), pp. 191-196.

21. Razavi, S., Emadzadeh, B., Rafe, A., \& Mohammad A. A. (2007). The physical properties of pistachio nut and its kernel as a function of moisture content and variety: Part I. Geometrical properties. Journal of Food Engineering. Vol.81. pp. 209-217. doi.10.1016/j.jfoodeng.2006.11.003.

22. Rijssenbeek, W. (2007). Jatropha Global Position. Workshop EU Brussels. RR Energy for FACT Foundation. p37

23. Shivani, P., Khushbu, P., Faldu, N., Thakkar V., \& Subramanian R. B. (2011). Extraction and analysis of Jatropha curcas L. seed oil. Afr J Biotechnol Vol.10(79) pp. 18210-18213

24. Sidibe Sayon. (2011). Contribution à l'étude des huiles végétales de coton et de Jatropha curcas comme biocarburant dans les moteurs diesels à injection directe, Fondation 2iE-Université de Ouagadougou, $234 \mathrm{p}$. 
25. Sirisomboon, P., Kitchaiya, P., Pholpho, T., \& Mahuttanyavanitch, W. (2007). Physical and mechanical properties of Jatropha curcas L. fruits, nuts and kernels. Biosyst. Eng. Vol. 97(2), pp. 201-207

26. Sofalne, C., Kengne, B., Carisey, N., Larochelle, F., Wehnelt, T. L., Yedmel, S., \& Fokam, M. G. (2016). Plan d'actions forestier ABIOGet 2016-2020, PAFA, pp 55

27. Tangermann S., (2007). Biocarburants et sécurité alimentaire, Économie rurale 300, , pp 100-104. doi : 10.4000/economierurale. 2260

28. Tavakoli, H., Mohtasebi, S.S., \& Jafari, A. 2009. Effect of Moisture Content and Loading Rate on the Shearing Characteristics of Barley Straw by Internode Position. Agricultural Engineering International: the CIGR Ejournal. Manuscript 1176. Vol. 11.

29. Tchobsala, (2008). Inventaire des variétés de Jatropha dans la zone cotonnière du nord Cameroun, Rapport de la mission conjointe entre la SODECOTON et l'IRAD du 13 au 26 mars, p34.

30. Vaitilingom, G. (2007). Extraction, Conditionnement et utilisation des huiles végétales pures carburant. Montpellier, France, octobre 2007. In Conférence Internationale « Enjeux et Perspectives des Biocarburants pour l'Afrique ». Ouagadougou, Burkina Faso, p52.

31. Vursavus, K., \& Ozguven, F. (2004) Mechanical behaviour of apricot pit under compression loading, J. Food Eng., Vol. 65, pp. 255-261.

32. Zavrajnov, A. I., \& Nikolow, D. I. (1990). Mechanization of the preparation and the forage conservation. Vol 3. pp. 336. ISBN-5-10000761 